\title{
Task Analysis of Paramedics in the Ambulance Patient Compartment
}

\author{
Rosnah Mohd. Yusuff ${ }^{1,}$, , Abdul Malik Bin Zainal Abidin ${ }^{2, b}$ \\ and Fazlollah Agamohamadi ${ }^{3, c}$ \\ ${ }^{1,2,3}$ Department of Mechanical and Manufacturing Engineering, Engineering Faculty \\ University Putra Malaysia \\ a Rosnah@eng.upm.edu.my, ${ }^{\mathrm{C}} \mathrm{f}$ _agamohamadi@yahoo.com
}

Keywords: Ambulance, patient compartment, paramedic safety, task analysis

Abstract. An ambulance is designed through the modification of an existing standard vehicle body. By converting, renovating and equipping a standard vehicle body with a patient stretcher, paramedic seats, nearside seats, and related medical equipment, the vehicle becomes an ambulance. Therefore, the requirements and layout of the ambulance interior are constrained by the space available and dimensions of the adopted vehicle. Ambulance occupant protection, safety and ergonomic aspects are usually compromised. High quality and consistent emergency care demand continuous quality improvement and is directly dependent on the effective monitoring, integration, and evaluation of all components of the patient's care. Currently, there is no standard or guideline regarding patient compartment layout to help ambulance manufacturers to improve this confine working space. This study aims to assess and evaluate ambulance patient compartment and its effect on the paramedics in performing their tasks. Since the paramedics have to respond to emergencies, their comfort, safety and ease of handling the various equipment in the ambulance has to be considered. A combination of techniques was applied to collect comprehensive data, including interviews, observations and questionnaires which outcomes are used as a basis of suggestions for the improvement in ambulance layout design. By analyzing the tasks performed, a proper layout which considers the ergonomic aspects will ensure that the first response is efficient and reliable.

\section{Introduction}

Ambulance is a specialized vehicle use in pre hospital service, built and equipped for a special application. The design, construction, accommodation, and safety of the patient compartment are important to ensure this first response service is efficient and reliable.

Usually a standard van body is converted to an ambulance by renovating and equipping it with a patient stretcher, paramedic seats, nearside seats, and related medical equipment. The design of an ambulance patient compartment is a complex challenge with design options constrained by space limitations and the requirements of emergency driving. As the space is tightly confined, the patient compartment is difficult to design because altering the specification to support one clinical task will affect other areas of equipment placement and storage. The design challenges reflect the current experience and working environment of ambulance personnel. There is no international standard for designing or arranging the patient compartment layout [1].

This is particularly true of ambulances used by the Ministry of Health in Malaysia. No studies have been done on the effect of the ambulance design on the performance of Malaysian paramedics. The comfort, safety, and ease of handling of equipment in the ambulance compartment for Malaysian paramedics have never been investigated. The study will be carried out for the van (standard body) because Malaysia currently uses fleets of automotive vans, designed and manufactured with specialized aftermarket additional retrofits to adapt them to the ambulance market. Four existing ambulance chassis model which are used as Advance Life Support (ALS) ambulance were involved in this study.

This study aims to highlight some of these issues by identifying the tasks carried out by the paramedics and the difficulties they faced in terms of clearance and reach-ability of essential equipment during their tasks. This analysis is an important input in designing the layout of the ambulance compartment. The stakeholders consisting of doctors, paramedics, medical assistance, engineers and ambulance drivers were considered. 
Background of study. The paramedic's work environment is largely unpredictable and is well established as a source of considerable stress [2]. Much of the paramedic's time is spent providing patient care while transporting one or more patients in the patient compartment of the ambulance. The confined space of the patient compartment, the task requirements, and the configuration of equipment within the patient compartment contribute to risk of musculoskeletal injury (MSI) by requiring the paramedic to adopt and maintain awkward postures [3]. As with other health care providers, the requirements for patient handling present a significant risk of injury to paramedics [4, 5]. The interface between the ambulance and loading system is also one of the activities that contribute to Musculoskeletal disorders (MSDs) problem.

Lavender, et al. [5] analyzed the biomechanical stresses placed on the body based on simulations of frequently performed tasks. The relative risk of low back disorders was quantified using a trunk motion model. The hazardous tasks performed included pulling a victim from bed to stretcher, the initial descent of stairs using a stretcher, and lifting a victim on a back board from the floor. The data indicated where engineering changes to equipment regularly used by ambulance personnel would have the greatest impact in reducing the risk of MSI.

Harmful postures are a feature of working in an ambulance. Doormaal, et al. [6] assessed tasks using the Ovako Working posture Analysis System (OWAS) and the Work and Health questionnaire. A biomechanical model was applied to several specifically strenuous simulated conditions. The results showed that $16-29 \%$ of a work shift was spent in a harmful posture The recommendations included issues around the equipment in ambulance, training of ambulance assistants and adaptations of working procedures, including the position of the attendant to the side and not at the head of the patient.

Nine design challenges have been identified by The National Patient Safety Agency, The Helen Hamlyn Research Centre, [7] to provide a basis for future evidence-based standardization. Significant improvements in safe and effective working environment will be delivered by solving these challenges in a consistent way across the full range of vehicle used now, and in the future. This will also enhance patient safety, patient experience/satisfaction, and facilitate cost-efficient purchasing and procurement. The challenges are:

1. Ensure safe and effective access and egress

2. Improve working space and layout

3. Effectively secure people and equipment in transit

4. Ensure effective communication

5. Address security, violence and aggression

6. Facilitate effective hygiene and infection control

7. Maximize equipment usability and compatibility

8. Improve vehicle engineering

9. Humanize the patient experience

\section{Material and methods}

A combination of techniques was chosen to gather comprehensive data about the workspace and the patient care tasks performed including interviews, observations and questionnaires. Outcome from the observation, questionnaire and interview can be used as a basis for the suggestion for the improvement in ambulance layout design.

Interview. The interview guides were tailored to the different stakeholder groups, but all focused on identifying the strengths and weaknesses of the current patient compartment. Questions from the engineers and medical staff interviewed include the following:

- Malaysian policies in providing standard ambulance

- Aspects of equipment and layout standardization

- Procedure in providing care in ambulance

- Infection control 
- Ambulance technical specification

- Comfort

- Medical equipment and consumable items

Questionnaire. A questionnaire on evaluation of performance were distributed to paramedics and medical staff by sending copies to Hospital Putrajaya, Hospital Serdang, Hospital Kuala Lumpur and Hospital Selayang. The number of musculoskeletal complaints was recorded base on modified Nordic Body Map Questionnaire.

\section{Results and Discussion}

The data gathered from observation, interviews, questionnaire, and task simulation were analyzed and discussed. Information from literature and findings from existing layout will be integrated to propose the improved layout.

Questionnaire and Survey. Respondents for the questionnaire were 30 medical staff with 24 males and 6 females from Hospital Kuala Lumpur, Hospital Serdang, Hospital Selayang, and Hospital Putrajaya. The paramedics or medical assistants spent an average $5.6 \mathrm{~h}(70 \%)$ of an $8 \mathrm{~h}$ shift in the patient compartment. The information was collected by visiting the Emergency Department at these hospitals. They were asked to rate the adequacy of features in the patient compartment, within the context of performing their job safely. All the information was gathered either by questionnaire or interview session. Ranking of the most frequently occurring clinical tasks were:

- Check pulse/blood oxygen saturation (93\% of calls)

- Check blood pressure (93\% of calls)

- Administer oxygen ( $73 \%$ of calls)

Within the ambulance (Table 1), the most physically demanding activity reported by paramedics was performing cardiopulmonary resuscitation (CPR), which is inherently demanding due to the requirement for chest compressions. Paramedics also identified the effort required to access the patient and equipment within the ambulance as physically demanding.

Table 1: Physically demanding Tasks of managing patients in ambulance

\begin{tabular}{|l|c|}
\hline \multicolumn{1}{|c|}{ Physically demanding aspect } & Number of respondents \\
\hline CPR & 24 \\
\hline Accessing patient (e.g., right side, legs) & 18 \\
\hline Accessing equipment (e.g., under bench, across patient) & 17 \\
\hline Seating (e.g., bench high, seat belts, too deep, sideways) & 15 \\
\hline Ride quality/bracing & 12 \\
\hline$>1$ patient/ transfer of secondary stretcher to swivel seats & 10 \\
\hline Confined space (e.g., head room, foot space, moving in vehicle) & 7 \\
\hline Bending over (unspecified context) & 6 \\
\hline Monitoring vitals & 6 \\
\hline I.V. & 5 \\
\hline Vomiting/ tuming patient & 3 \\
\hline Writing (e.g., while in motion, no writing surface) & 3 \\
\hline Bag valve mask & 2 \\
\hline Repositioning patient & 2 \\
\hline Communication / noise levels & 2 \\
\hline Intubation & 2 \\
\hline
\end{tabular}

The level of effort required to perform tasks within the ambulance was perceived to be greatest for CPR, loading the stretcher, writing while the vehicle is in motion, and working from the jump seat (Table 2). 
Table 2: Mean level of effort required to perform tasks within the ambulance

\begin{tabular}{|l|c|}
\hline \multicolumn{1}{|c|}{ Physically demanding aspect } & $\begin{array}{c}\text { Level of Effort } \\
\text { (1= very easy; } \mathbf{5} \text { very difficult })\end{array}$ \\
\hline CPR* & 4.0 \\
\hline Loading stretcher & 3.8 \\
\hline Writing* & 3.1 \\
\hline Working from the jump seat* & 2.9 \\
\hline Intubating or initiating I.V. & 2.8 \\
\hline Working from the bench seat* & 2.7 \\
\hline Monitoring patient status* & 2.6 \\
\hline Accessing cup boards* & 2.2 \\
\hline Removing portable oxygen & 2.1 \\
\hline Removing monitors & 1.9 \\
\hline Raising head/ back rest* & 1.8 \\
\hline Removing the jump kit & 1.7 \\
\hline
\end{tabular}

Note: * indicates tasks performed while vehicle is in motion

The paramedics and medical assistants rated the noise $(63.3 \%$ poor and very poor) and vibration levels $(60.0 \%$ poor and very poor) in patient compartment to be high. Medical staff rated poor and very poor the overall space $(60.0 \%)$, overhead clearance $(40.0 \%)$, access to the patient $(43.3 \%)$, line of sight to patient $(53.3 \%)$, ability to access equipment while seated $(50.0 \%)$, access equipment while standing (46.7\%), access to jump kit (56.7\%) and arrangement of storage/equipment (56.7\%). Majority of the staff had concerns regarding the overall space, line of sight to patient, access to equipment, access to the jump kit, and arrangement of storage/equipment. Table 4 provides a summary of the number of respondents indicating each rating category for the design features of the patient compartment.

Table 4: Rating of features within the patient compartment

\begin{tabular}{|l|c|c|c|c|c|}
\hline & Verv poor & Poor & Good & Very good & Excellent \\
\hline Environment & & & & & \\
\hline Lighting & 0 & 12 & 15 & 2 & 1 \\
\hline Noise & 1 & 18 & 10 & 1 & 0 \\
\hline Vibration & 4 & 14 & 10 & 2 & 0 \\
\hline Layout & & & & & \\
\hline Overall space & 4 & 14 & 10 & 2 & 0 \\
\hline Overhead clearance & 1 & 11 & 16 & 2 & 0 \\
\hline Access to patient & 1 & 12 & 16 & 1 & 0 \\
\hline Line of sight to patient & 2 & 14 & 13 & 1 & 0 \\
\hline Access to equipment while seated & 3 & 12 & 14 & 1 & 0 \\
\hline Access to equipment while standing & & 14 & 15 & 1 & 0 \\
\hline Access to jump kit & 1 & 16 & 12 & 1 & 0 \\
\hline Arrangement of storage/equipment & 0 & 17 & 12 & 1 & 0 \\
\hline Seating & & & & & 0 \\
\hline Location & 2 & 11 & 16 & 1 & 0 \\
\hline Comfort & 1 & 7 & 19 & 3 & 0 \\
\hline Back support & 2 & 13 & 12 & 3 & 0 \\
\hline Handles, Latches, Knobs & & & & & 0 \\
\hline Ease of opening & 1 & 11 & 15 & 3 & 0 \\
\hline Comfort & 1 & 9 & 17 & 2 & 0 \\
\hline Within reach & 0 & 9 & 18 & 3 & 0 \\
\hline Adequate number of handheld & 0 & 7 & 19 & 4 & 0 \\
\hline
\end{tabular}

The data collected from the modified Nordic Body Map Questionnaire showed as in Table 5 that the major complain for critical musculoskeletal pains are on the neck (71.4\%) and lower back (89.3\%), and $50.0 \%$ in the right shoulder.

Loading/Unloading. The most hazardous activity identified was loading the ambulance stretcher and patient onto the ambulance. The ambulance stretcher height is not easily adjustable and the wheels are unsuitable for long-distance travel within the hospital. Literatures showed that the easy loader, winch \& ramp, and tail lift were the most popular methods of lifting used.

The easy-loader stretcher had the highest REBA score (8.1) followed by the tail lift (5.8) and the ramp and winch (5.7). It was found that the easy-loader scored significantly higher than all of the other systems. The risk level using the easy-loader was high with action category of 3 (action is 
necessary soon). For the other systems the risk level was medium with an action category of 2 (action is necessary). NIOSH was used to validate the REBA analysis [8].

Table 5: The number of complaints for each body part

\begin{tabular}{|c|c|c|c|c|}
\hline \multirow{2}{*}{ No } & \multirow{2}{*}{\multicolumn{2}{|c|}{ Part of the body (Location) }} & \multicolumn{2}{|c|}{ Complaint } \\
\hline & & & No of participant & $\%$ \\
\hline 1 & Neck & - & 20 & 71.4 \\
\hline 2 & \multirow{2}{*}{ Shoulder } & Right & 14 & 50.0 \\
\hline 3 & & Left & 12 & 42.9 \\
\hline 4 & \multirow{2}{*}{ Upper Back } & Right & 12 & 42.9 \\
\hline 5 & & Left & 8 & 28.6 \\
\hline 6 & \multirow{2}{*}{ Upper Arm } & Right & 10 & 35.7 \\
\hline 7 & & Left & 8 & 28.6 \\
\hline 8 & Lower Back & - & 25 & 89.3 \\
\hline 9 & \multirow{2}{*}{ Forearm } & Right & 4 & 14.3 \\
\hline 10 & & Left & 5 & 17.9 \\
\hline 11 & \multirow{2}{*}{ Wrist } & Right & 5 & 17.9 \\
\hline 12 & & Left & 5 & 17.9 \\
\hline 13 & \multirow{2}{*}{ Hand } & Right & 5 & 17.9 \\
\hline 14 & & Left & 5 & 17.9 \\
\hline 15 & Hip/Buttocks & - & & \\
\hline 16 & \multirow{2}{*}{ Thigh } & Right & 8 & 28.6 \\
\hline 17 & & Left & 8 & 28.6 \\
\hline 18 & \multirow{2}{*}{ Knee } & Right & 11 & 39.3 \\
\hline 19 & & Left & 9 & 32.1 \\
\hline 20 & \multirow{2}{*}{ Lower Leg } & Right & 7 & 25.0 \\
\hline 21 & & Left & 7 & 25.0 \\
\hline 22 & \multirow{2}{*}{ Foot } & Right & 12 & 42.9 \\
\hline 23 & & Left & 12 & 42.9 \\
\hline
\end{tabular}

Results of Interviews. Findings from discussion with respondents consist of working procedure, and comfort used as the basis of information for further investigation and recommendation. Activities that can contribute to the risk of injuries and musculoskeletal complain by users were identified.

Work Procedures.Ambulance services must carry equipment and medications as per requirement of Treatment Protocols, Ambulance services should not equip ambulances with equipment that is outside of scope of practice of its Emergency Medical Technician (EMT) employees. All equipment must be designed and constructed to meet medical performance objectives and must not endanger patients. In the new ambulances design such that the requirement of ambulance operators such as the accessibility to any equipment is within reach. The 'ergonomic layout concept' means that allnecessary facilities including equipment are accessible without much hassle.

Comfort. The clearances between the seating paramedics and the head of the patient must be defined and established. Besides, the clearances between the swivel chairs and the stretcher must allow for appropriate space for easy movement of people when required. The position of chairs is often debated on whether it should be fixed or a bench. During travelling, some ambulance operators prefer to sit facing forward rather than facing the patient.

Risk of Injuries. The greatest risk of injury exists when there is extreme exposure to any single risk factor, or when more than one risk factor occurs simultaneously [9]. The primary risk factors for MSI were identified and summarized relative to specific awkward postures that were commonly observed.

Forward flexion of the trunk occurs in combination with rotation (twisting) and high forces during many activities that are common for paramedics. Flexion in excess of 90 degrees is often sustained for long periods in load-bearing positions. These factors present risk of injury to the lower back. Sitting postures within the patient compartment are generally unsupported due to the requirement to maintain proximity to the patient or equipment, and due to the height and depth of the seat. Flexion of the trunk is often accompanied by extension and rotation of the neck to maintain a forward visual field, which presents a risk of injury to the neck. Static extension of the neck in excess of 45 degrees accompanied by rotation in excess of 30 degrees was observed. Activities observed during task simulations that are associated with risk factors for low back injury are listed in Table 6. 


Table 6: Activities presenting risk to the lower back and risk factors
\begin{tabular}{|l|l|}
\hline \multicolumn{1}{|c|}{ Activity } & \multicolumn{1}{c|}{ Concurrent Risk Factors } \\
\hline Sitting (e.g., at station, waiting, in transit ) & Prolonged, static flexion; rotation, vibration \\
\hline Raising and lowering stretcher & High force, flexion \\
\hline Raising stretcher wheels & Moderate forces; rotation, flexion, lateral flexion \\
\hline Loading/unloading stretcher & High force \\
\hline CPR & High force; flexion; repetition; static posture \\
\hline Monitoring vital signs & Static flexion, rotation while seated \\
\hline Reaching equipment in cupboards & High force (bracing); flexion, rotation \\
\hline $\begin{array}{l}\text { Reaching far side of patient (e.g., patient straps, dressing } \\
\text { wounds, cutting clothes) }\end{array}$ & High force (bracing); flexion, rotation \\
\hline Bag valve mask & Static posture; flexion \\
\hline Accessing jump kit/ALS kit on seat beside paramedic & Rotation; lateral flexion; high forces (bracing) \\
\hline
\end{tabular}

Shoulder flexion and abduction occurs in combination with high forces and could be considered repetitive due to the range in activities that result in flexion greater than 45 degrees. Extreme postures of the shoulder (flexion and abduction greater than 90 degrees) that are often maintained for more than 30 seconds are utilized while using grab bars to brace against motion inside the vehicle or when accessing equipment in the cabinets. Removal of jump kits, monitors and portable oxygen from the ambulance require extreme postures, often combined with ballistic forces to jerk equipment into position. Loading the main stretcher into the ambulance requires that the bottom of the stretcher is lifted clear of the floor. This was observed to require a forceful shoulder elevation for paramedics of shorter stature or when the ambulance was parked on an incline. These factors present a risk of injury to the shoulder and upper back. Activities observed during task simulations that are associated with risk factors for injury to the shoulder or upper back are listed in Table 7.

Table 7: Activities presenting risk to shoulders or upper back and concurrent risk factors

\begin{tabular}{|l|l|}
\hline \multicolumn{1}{|c|}{ Activity } & \multicolumn{1}{c|}{ Concurrent Risk Factors } \\
\hline Loading stretcher into ambulance & High forces, shoulder elevation \\
\hline Releasing stretcher mount & High impact forces, flexion \\
\hline Reaching equipment in cupboards & High force, extreme flexion and abduction \\
\hline Bracing against motion (holding rails) & High unpredictable forces; static flexion and abduction \\
\hline Removing jump kits & High ballistic forces; flexion or extension and abduction \\
\hline Removing monitors & High ballistic forces; flexion \\
\hline Removing portable oxygen & High ballistic forces; shoulder elevation and abduction \\
\hline CPR & High forces; repetitive; static flexion \\
\hline Raising head of stretcher from bench seat & High forces; flexion of right shoulder \\
\hline Tending to patient (vitals, inspection, etc.) & Static posture; flexion; occasional high forces \\
\hline Bag valve mask & Static flexion (may be supported by knees) \\
\hline
\end{tabular}

Petzall [10] and Ferreira and Hignett [11]discuss access/egress issues with respect to the design of doorways and the physical task of loading and unloading. Most of these issues were identified in the comparison of the three stretcher loading system.

The patient compartment layout of the ambulance and the time spent working in this confined space, added to the fact that it is increasing, presents a risk to staff and patients. Medical equipment and consumable items all have the potential hazards in the patient compartment. Ferreira and Hignett [11] looked at the layout of the patient compartment and [6] looked at the effect of the layout on operator posture. The design challenge of space and layout are clear issues that need to be addressed particularly with respect to the working procedure, work system and how medical staff work in the ambulance.

The range of medical equipment and consumable items is large because most of them are loose items and it is suggested the interfaces between the equipment and the vehicle would benefit from standardization. Providing standard placement with suitable receptacles/restrains would be beneficial to ensure the safety, usability, portability, maneuverability, and hygiene.

\section{Conclusion and Recommendation}

This project reviewed the tasks of paramedics and the problems faced in ambulance with respect to clinical efficiency and paramedic postural safety. The most frequently occurring clinical tasks were checking pulse/blood oxygen saturation, checking blood pressure, oxygen administration, and check 
blood glucose concentration. The most physical demanding activity reported by paramedics and medical assistance was performing CPR. The level of effort required to perform CPR perceive to be greatest, followed by loading the stretcher, writing while the vehicle is moving and working from jump seat. The questionnaire analysis provided information on discomfort, and perceptions regarding causation of discomfort and injury and the design of the ambulance and patient compartment layout.

Additional research is needed about the tasks performed and postures adopted by paramedics for patient loading and unloading, treatment of critical patients and major disaster scenarios. A proper understanding of the safety and ergonomics of this work place would assist greatly in enhancing and optimizing ground ambulance vehicle design and safety for effective patient care during transport, and may facilitate in creating an environment where there is a decreased perceived need for risky driving practice.

\section{REFERENCES}

[1] J. Seary, et al., "Healthcare quality and ergonomics, evidence based design, safety by design," 2007.

[2] K. M. Young and C. L. Cooper, "Occupational stress in the ambulance service: a diagnostic study," Health and Manpower Management, vol. 23, pp. 140-147, 1997.

[3] J. Letendre, et al., "Title," unpublished|.

[4] S. A. Lavender, et al., "Postural anslysis of paramedics simulating frequently performed strenuous work tasks," Applied Ergonomics, vol. 31, pp. 167-177, $2000 \mathrm{~b}$.

[5] S. A. Lavender, et al., "Postural analysis of paramedics simulating frequently performed strenuous work tasks," Applied Ergonomics, vol. 31, 2000a.

[6] M. T. A. J. Doormaal, et al., "Physical workload of ambulance assistants," Ergonomics, vol. 38, pp. 361-376, 1995.

[7] The Helen Hamlyn Research Centre-The Royal College of Art and The National Patient Safety Agency, "Design for patient safety-Future ambulances," ed. UK: London, 2007.

[8] S. Hignett and E. Ghassemieh, "Design and performance of ambulance stretcher loading systems," vol. 125,307 (£), ed. Ergonomics (Human Sciences)- Loughborough University: East Anglian Ambulance NHS Trust, East Midlands Ambulance Service NHS Trus, London Ambulance NHS Trust, Two Shires Ambulance NHS Trust, 2006.

[9] B. P. Bernard and L. Fine, "Musculoskeletal Disorders and Workplace Factors: A Critical Review of Epidemiological Evidence for Work-Related Musculoskeletal Disorders of the Neck, Upper Extremity and Low Back," National Institute for Occupational Safety and Health, U.S. Department of Health and Human Services, DHHS (NIOSH) Publication, No.97-141, 1997.

[10] J. Petzall, "The design of entrances of taxis for elderly and disabled passengers. An experimental study," Applied Ergonomics, vol. 26, pp. 343-352, 1995.

[11] J. Ferreira and S. Hignett, "Reviewing ambulance design for clinical efficiency and paramedic safety," Applied Ergonomics, vol. 36, pp. 97-105, 2005. 